\title{
Grain-boundary melting: A Monte Carlo study
}

\section{Besold, Gerhard; Mouritsen, Ole G.}

\section{Published in:}

Physical Review B

Link to article, DOI:

10.1103/PhysRevB.50.6573

Publication date:

1994

\section{Document Version}

Publisher's PDF, also known as Version of record

Link back to DTU Orbit

\section{Citation (APA):}

Besold, G., \& Mouritsen, O. G. (1994). Grain-boundary melting: A Monte Carlo study. Physical Review B, 50(10), 6573-6576. https://doi.org/10.1103/PhysRevB.50.6573

\section{General rights}

Copyright and moral rights for the publications made accessible in the public portal are retained by the authors and/or other copyright owners and it is a condition of accessing publications that users recognise and abide by the legal requirements associated with these rights.

- Users may download and print one copy of any publication from the public portal for the purpose of private study or research.

- You may not further distribute the material or use it for any profit-making activity or commercial gain

- You may freely distribute the URL identifying the publication in the public portal

If you believe that this document breaches copyright please contact us providing details, and we will remove access to the work immediately and investigate your claim 


\title{
Grain-boundary melting: A Monte Carlo study
}

\author{
Gerhard Besold \\ Lehrstuhl für Festkörperphysik, Universität Erlangen-Nürnberg, Staudtstrasse 7, D-91058 Erlangen, Germany \\ and Department of Physical Chemistry, The Technical University of Denmark, Building 206, DK-2800 Lyngby, Denmark \\ Ole G. Mouritsen \\ Department of Physical Chemistry, The Technical University of Denmark, Building 206, DK-2800 Lyngby, Denmark
}

(Received 1 February 1994)

\begin{abstract}
Grain-boundary melting in a lattice-gas model of a bicrystal is studied by Monte Carlo simulation using the grand canonical ensemble. Well below the bulk melting temperature $T_{m}$, a disordered liquidlike layer gradually emerges at the grain boundary. Complete interfacial wetting can be observed when the temperature approaches $T_{m}$ from below. Monte Carlo data over an extended temperature range indicate a logarithmic divergence $w(T) \sim-\ln \left(T_{m}-T\right)$ of the width of the disordered layer $w$, in agreement with mean-field theory.
\end{abstract}

One of the competing processes in polycrystalline solids by which the excess free energy of grain boundaries can be reduced is grain-boundary (or interfacial) melting: at temperatures below the bulk melting temperature $T_{m}$, a disordered, liquidlike layer gradually appears at the interface and its width diverges when $T_{m}$ is approached from below. ${ }^{1-3}$ Interfacial melting is a special case of "complete wetting,"3-6 and is related to "interfacial adsorption" as observed in simulations of multistate models with first-order bulk transitions. ${ }^{7}$ Theoretical predictions concerning interfacial melting differ depending on the framework of theoretical description (mainly Landau/mean-field theory), the range of interactions, and the spatial dimension of the system (for reviews see Refs. 3-5). The experimental characterization of interfacial and grain-boundary melting (in contrast to a related phenomenon, surface melting ${ }^{8}$ ) is in a somewhat unsatisfactory state mainly due to the extremely narrow range of temperatures over which the effect appears. ${ }^{9}$ This stresses the importance of performing computer experiments on model systems with well-defined dimensionality and interactions. Molecular-dynamics simulations of both two- and three-dimensional (2D) and (3D) LennardJones matter ${ }^{10}$ and of particles interacting via a Morse potential $^{11}$ dealt with grain-boundary melting of the interface between a few grains in constrained equilibrium. Only a single Monte Carlo study was concerned with nonequilibrium domain growth and interfacial melting in a multidomain configuration. ${ }^{12}$

In this paper, we present results from Monte Carlo (MC) simulation of constrained-equilibrium grainboundary melting of a 2D bicrystal (two-domain system) in a square lattice-gas model. The same model has already been studied by Kikuchi and $\mathrm{Cahn}^{13}$ using a meanfield-like cluster-variation method. These authors found a logarithmic divergence of the width of the liquidlike layer, $w(T) \sim-\ln \left(T_{m}-T\right)$, in agreement with general predictions from the Landau theory for systems with short-range interactions. ${ }^{3-5,14}$ In terms of effective interactions, the logarithmic divergence of the wetting lay- er can be explained by assuming an exponential repulsion between its two solid-liquid interfaces due to the overlap of their exponentially decaying density profiles within the liquidlike layer. ${ }^{13,15}$ However, a power law $w(T) \sim\left(T_{m}-T\right)^{-1 / 3}$ is predicted for short-range interactions in two dimensions if solid-liquid interface fluctuations are taken into account (details are reviewed, e.g., in Ref. 5). Therefore, a study of the same model with a method beyond mean-field theory may provide instructive results.

In the lattice-gas model studied here, occupation variables $c_{i} \in\{0,1\}$ are assigned to the $N$ sites of a square lattice (with lattice constant $a$ ) and coupled by the grand canonical lattice-gas Hamiltonian

$$
\mathscr{H}=-\sum_{n=1}^{5} \varepsilon_{n} \sum_{\langle i, j\rangle_{n}} c_{i} c_{j}-\mu \sum_{i=1}^{N} c_{i}
$$

where $\mu$ is the chemical potential and the $\varepsilon_{n}(1 \leq n \leq 5)$ are interaction parameters for pairs of occupied $n$ th-nearest-neighbor sites $\langle i, j\rangle_{n}$. By choosing $\varepsilon_{1}=\varepsilon_{2}=\varepsilon_{3}=-\infty$ (repulsive hard-core interaction) and $\varepsilon_{4}, \varepsilon_{5}>0$ (attractive interactions) with $\varepsilon_{4}=1.2 \varepsilon_{5}$, we obtain the model considered by Kikuchi and Cahn. ${ }^{13}$ The phase diagram of the model is known from transfermatrix calculations ${ }^{16}$ and it resembles that of a simple fluid system: it exhibits disordered "lattice-gas" and "lattice-liquid" phases, and a single crystalline "solid" phase with a first-order melting curve. The "solid" phase has a " $(\sqrt{5} \times \sqrt{5}) R \pm \arctan \frac{1}{2}$ " superstructure (which in surface physics is known, e.g., from alkali adsorbate systems ${ }^{17}$ ) with domains of different sign of the rotation angle of the square superstructure unit mesh with respect to the underlying square lattice (see Fig. 1). The hard-core repulsion can be visualized in terms of a hard-core disk of "atomic" diameter $\sqrt{5} a$ assigned to each occupied site (see Fig. 1).

The bicrystal configuration is now defined as follows: A rectangular-shaped strip with $2 M L$ lattice sites is cut off the square lattice with the short edge (length $M$ ) in the 


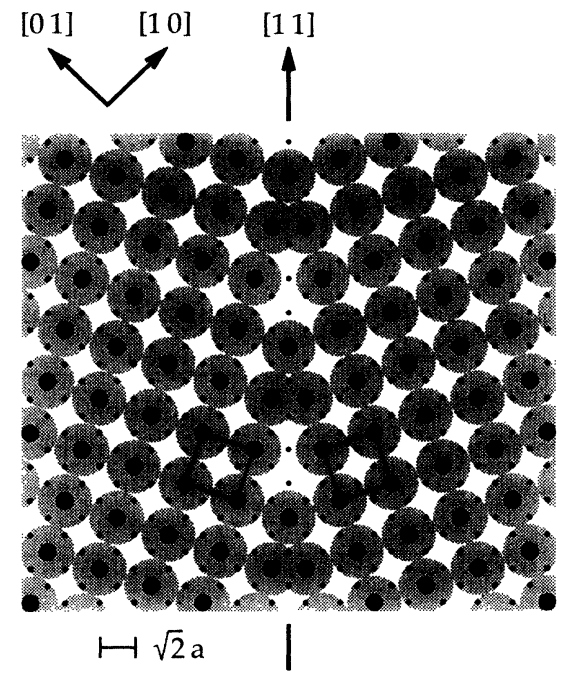

FIG. 1. Two-dimensional bicrystal on a square lattice with a high-angle symmetric tilt boundary in the [1 11$]$ direction ("unrelaxed" state). The figure shows the sites of the square lattice (dots), the occupied sites (solid circles), and the associated "atomic" hard-core disks with diameter $\sqrt{5} a$ (gray circles). Also shown are the unit cells of crystalline $(\sqrt{5} \times \sqrt{5}) R \pm \arctan \frac{1}{2}$ domains of type "+" (left) and "-" (right).

[1 1 ] direction and the long edge (length $L$ ) in the [1 $\overline{1}$ ] direction. (Here and in the following, all references to crystallographic directions refer to the underlying square lattice; $\sqrt{2} a$ is used as unit of the length scale.) Then the left half side of the lattice strip is occupied with a solid domain of type " + " and the right half side with one of type "-", leading to a high-angle $\left(36.87^{\circ}\right)$ symmetric tilt boundary in the [1 1 ] direction as shown in Fig. 1. This "unrelaxed" configuration still contains forbidden pairs of overlapping hard-core disks along the interface. By removing either site of each of these pairs, "relaxed" lattice configurations result which were used as start configurations of $\mathrm{MC}$ runs.

In order to prevent fluctuation-induced reorientation of the domains close to $T_{m}$, the occupation variables within columns of width 2 at both the left and right system boundary were clamped at their initial values during the simulation. This corresponds to a lattice strip of size $(L-4) \times M$ with free boundary conditions and staggered boundary chemical potentials of finite strength at the short edges. Periodic boundary conditions were imposed at the long edges. Kikuchi and Cahn used $(L, M)=(5,81)$, free boundary conditions throughout, and the boundary fields were defined in a slightly different way. ${ }^{13}$

Lattice strips of different size were considered in our MC simulations, but in this paper we only present the main results for the largest system size $L \times M$ $=100 \times 30$ (6000 lattice sites). Details will be discussed elsewhere. ${ }^{18}$ The simulations were performed at constant chemical potential $\mu / \varepsilon_{5}=-1.5$ using Glauber dynamics. After equilibration at a given temperature, up to 400000 MCS (Monte Carlo steps per site) were used for the calcu- lation of thermal averages.

Figure 2 displays a series of typical equilibrium microconfigurations for increasing temperature $T$. Up to $T \approx 0.5$ (in units of $\varepsilon_{5} / k_{B}$ ) only roughening of the grain boundary is observed. Above $T \approx 0.5$, a disordered, liquidlike layer appears at the interface and becomes broader with increasing temperature. The interfacial layer still shows a high degree of short-range order. In the temperature range $0.72 \lesssim T \lesssim 0.74$, the average width of the liquid layer $w(T)$ increases from about $0.15 L$ to $0.75 \mathrm{~L}$, and also fluctuations of the two solid-liquid interfaces increase considerably. This gives a rough estimate for the location of the bulk melting temperature $T_{m}(\mu)$.

Figure 3 shows the coverage (density), $\theta$ $=\left\langle\sum_{i} c_{i}\right\rangle /[2 M(L-4)]$, as a function of temperature (the index "i" runs over all lattice sites except the ones with clamped occupation variables $c_{i}$ ). In the range $0.72 \lesssim T \lesssim 0.74, \theta(T)$ decreases rapidly with an inflection point at $T \approx 0.729$ which is taken as a better estimate for $T_{m}$.

Kikuchi and Cahn ${ }^{13}$ determined $w(T)$ from the excess entropy relative to a reference system without a grain boundary. In order to save the computer time needed for simulations of the reference system, another approach was used in this study. Parallel to the initial interface,
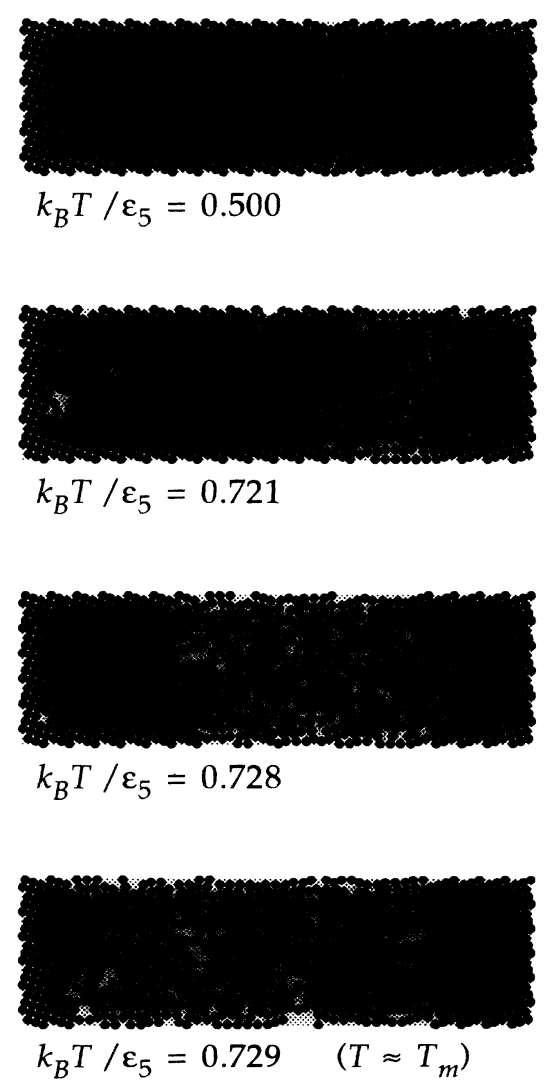

FIG. 2. Typical Monte Carlo microconfigurations of a bicrystal model of size $L \times M=100 \times 30$ (6000 lattice sites) after equilibration at the temperature $T$ shown below each picture $\left(\mu / \varepsilon_{5}=-1.5\right)$. Hard-core disks are indicated by solid circles. 


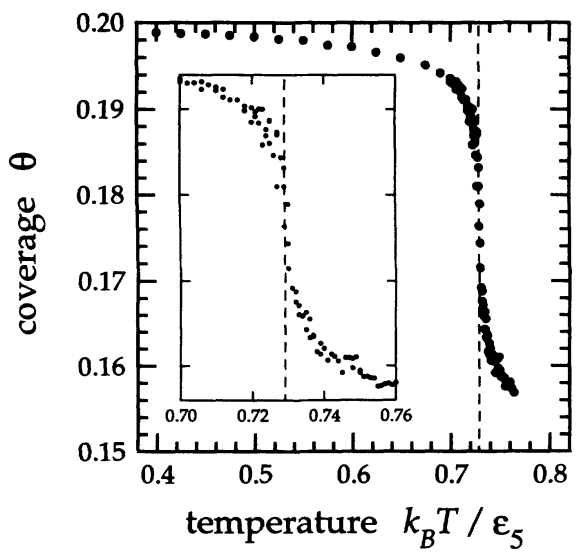

FIG. 3. Plot of the equilibrium coverage $\theta$ vs temperature $T$, at $\mu / \varepsilon_{5}=-1.5$ for the $L \times M=100 \times 30$ bicrystal. The dashed vertical line indicates the finite-system bulk melting temperature $T_{m} \approx 0.729 \varepsilon_{5} / k_{B}$. The inset displays the data points close to $T_{m}$ on an enlarged temperature scale.

the lattice strip was divided into $L=100$ columns. For each microconfiguration the average density per column was evaluated, resulting in a density profile normal to the interface direction. For each profile the center of gravity was then determined and shifted to the same position (column no. $L / 2=50$ ) before averaging the profiles. The ensemble averaged profiles determined in this way are shown in Fig. 4 as a function of temperature. When $T_{m} \approx 0.729$ is approached from below, the minimum of the density profiles drops and at $T \approx T_{m}$ a plateau at about $80 \%$ of the density of the "solid" phase emerges (cf. Fig. 4). Also the wings of the solid-liquid-interfaces now touch the system boundaries which indicates the onset of finite-size effects at or even slightly below $T_{m}$.

The full width at half minimum (FWHM) of the densi-

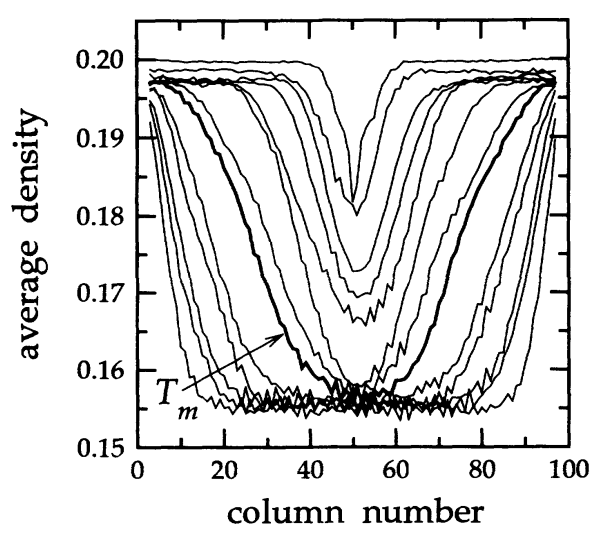

FIG. 4. Sequence of equilibrium density profiles normal to the grain-boundary direction for increasing temperature $T$. From top to bottom the profiles correspond to $k_{B} T / \varepsilon_{5}=0.5000$, $0.6500,0.7040,0.7160,0.7220,0.7260,0.7284,0.7290\left(T \approx T_{m}\right.$, bold profile), $0.7310,0.7330,0.7370,00.7400$, and 0.7500 . The data refer to simulations for the $L \times M=100 \times 30$ bicrystal model at $\mu / \varepsilon_{5}=-1.5$. Each profile was obtained by averaging over up to 4000 independent profiles. ty profiles is now taken as a measure for the average width of the interfacial layer $w(T)$. First a logarithmic law

$$
w(T)=-A \ln \left(T_{m}-T\right)+B,
$$

as expected from the mean-field theory, is fitted to the data points for $T \leq 0.729$, resulting in the best fit values $A=5.56 \pm 0.22, \quad B=-5.2 \pm 0.9, \quad$ and $\quad T_{m}=0.72903$ \pm 0.00003 . In particular the best-fit value for $T_{m}$ turns out to be quite stable (up to changes in the last decimal) even when some of the data points close to $T=0.729$ are excluded from the fit. Both the FWHM data as well as the best-fit function are shown in Fig. 5. Although the data points extend over four orders of magnitude of the reduced temperature $\left(T_{m}-T\right) / T_{m}$, the corresponding FWHM values cover only about 1.2 orders of magnitude. It is therefore not surprising that also a power law, $w(T)=C\left(T_{m}-T\right)^{-n}+D$ with parameters $C, D$, and $n$, can be fitted to the data with almost the same goodness of fit. However, the best-fit value for the exponent $n=0.05 \pm 0.04$ is so small that it is unlikely there could be a theoretical justification for it. In addition, $n$ depends sensitively on the data points used for the fit. Therefore there seems to be no support from the simulations for a power law to represent the entire set of FWHM data. The possibility remains of a crossover from a logarithmic to an asymptotic power law (due to a long-range entropic repulsion of the two fluctuating solid-liquid-interfaces ${ }^{3-6}$ very close to $T_{m}$, i.e., for $\left(T_{m}-T\right) / T_{m} \lesssim 10^{-5}$. Due to a lack of data points in this regime, however, this can be neither confirmed nor excluded on the basis of the available data.

In summary, our Monte Carlo simulations of grainboundary melting of a 2D bicrystal in constrained equilibrium confirm a logarithmic divergence of the width of the interfacial liquid layer, as predicted by mean-field theory. Most probably fluctuations are too suppressed in

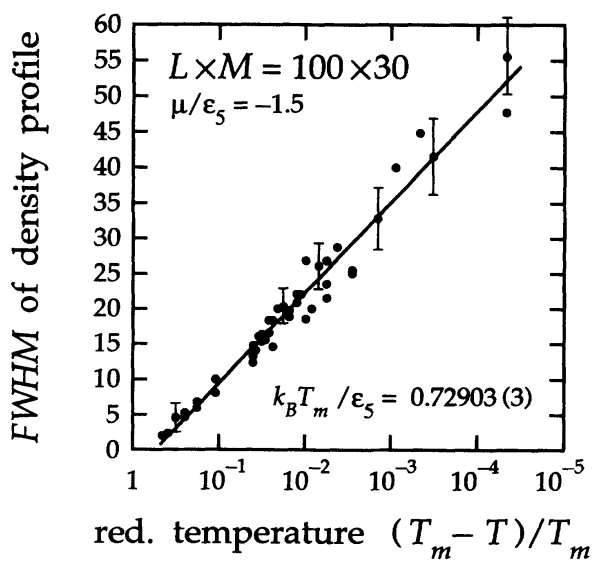

FIG. 5. Semilog plot of the width of the melted interfacial layer as given by the FWHM of density profiles (cf. Fig. 4) vs reduced temperature $\left(T_{m}-T\right) / T_{m}$. Error bars are given only for selected data points. The straight solid line is the result of a fit of the logarithmic law, Eq. (2), to the data. The resulting best fit value of the bulk melting temperature $T_{m}$ is indicated. 
the finite-size simulations to permit observation of a possible crossover to an asymptotic power-law behavior.

The authors would like to thank T. Gil, P. Lyngs Hansen, J. H. Ipsen, F. Yssing Hansen, L. Mikheev, and B. Reppich for stimulating discussions. G. B. was support- ed during his stays in Denmark by the Center for Modelling, Nonlinear Dynamics, and Irreversible Thermodynamics (MIDIT). O.G.M. acknowledges the support of the Canadian Institute of Advanced Research. The work was supported by the Danish Natural Science Research Council (11-0065-1).
${ }^{1}$ L. E. Murr, Interfacial Phenomena in Metals and Alloys (Addison-Wesley, Reading, MA, 1975), p. 321.

${ }^{2}$ L. K. Fionova and A. V. Artemyev, Grain Boundaries in Metals and Semiconductors (Les Éditions de Physique, Les Ulis Cedex, France, 1993), p. 211.

${ }^{3} \mathrm{~S}$. Dietrich, in Phase Transitions and Critical Phenomena Vol. 12, edited by C. Domb and J. L. Lebowitz (Academic, London, 1988), p. 1.

${ }^{4}$ D. E. Sullivan and M. M. Telo de Gama, in Fluid Interfacial Phenomena, edited by C. A. Croxton (Wiley, New York, 1986), p. 45.

${ }^{5} \mathrm{M}$. Schick, in Liquids at Interfaces, edited by J. Charvolin, J. F. Joanny, and J. Zinn-Justin (Elsevier, Amsterdam, 1990), p. 415.

${ }^{6}$ M. E. Fisher, J. Stat. Phys. 34, 667 (1984); M. E. Fisher, in Fundamental Problems in Statistical Mechanics VI, edited by E. G. D. Cohen (North-Holland, Amsterdam, 1985), p. 1; M. E. Fisher, J. Chem. Soc. Faraday Trans. 2, 82, 1569 (1986).

${ }^{7} \mathrm{~W}$. Selke, in Static Critical Phenomena in Inhomogeneous Systems, edited by A. Pekalski and J. Sznajd (Springer, Berlin, 1984), p. 191.

${ }^{8}$ J. G. Dash, Contemp. Phys. 30, 89 (1989).

${ }^{9}$ M. E. Glicksman and C. L. Vold, Surf. Sci. 31, 50 (1972).

${ }^{10}$ R. M. J. Cotterill, T. Leffers, and H. Lilholt, Philos. Mag. 30, 265 (1974); F. Carrion, G. Kalonji, and S. Yip, Scr. Metall. 17, 915 (1983); G. Ciccotti, M. Guillopé, and V. Pontikis,
Phys. Rev. B 27, 5576 (1983); G. Kalonji, P. Deymier, R. Najafabadi, and S. Yip, Surf. Sci. 144, 77 (1984); P. Deymier and G. Kalonji, J. Phys. (Paris) Colloq. 46, C4-213 (1985); P. Deymier and G. Kalonji, Scr. Metall. 20, 13 (1986); J. Q. Broughton and G. H. Gilmer, Phys. Rev. Lett. 56, 2692 (1986); J. F. Lutsko, D. Wolf, S. Yip, S. R. Phillipot, and T. Nguyen, Phys. Rev. B 38, 11572 (1988).

${ }^{11}$ P. S. Ho, T. Kwok, T. Nguyen, C. Nitta, and S. Yip, Scr. Metall. 19, 993 (1985); T. Nguyen, P. S. Ho, T. Kwok, C. Nitta, and S. Yip, Phys. Rev. Lett. 57, 1919 (1986).

${ }^{12}$ O. G. Mouritsen and M. J. Zuckermann, Phys. Rev. Lett. 58, 389 (1987).

${ }^{13}$ R. Kikuchi and J. W. Cahn, Phys. Rev. B 21, 1893 (1980).

${ }^{14}$ R. Lipowsky, Phys. Rev. Lett. 49, 1575 (1982); Z. Phys. B 51, 165 (1983); R. Lipowsky and W. Speth, Phys. Rev. B 28, 3983 (1983).

${ }^{15}$ A. A. Chernov and L. V. Mikheev, Phys. Rev. Lett. 60, 2488 (1988); A. A. Chernov and L. V. Mikheev, Physica A 157, 1042 (1989).

${ }^{16}$ J. Orban, J. Van Craen, and A. Bellemans, J. Chem. Phys. 49, 1778 (1968).

${ }^{17} \mathrm{~K}$. Müller, G. Besold, and K. Heinz, in Physics and Chemistry of Alkali Adsorption, edited by H. P. Bonzel, A. M. Bradshaw, and G. Ertl (Elsevier, Amsterdam, 1989), p. 65.

${ }^{18} \mathrm{G}$. Besold and O. G. Mouritsen (unpublished). 


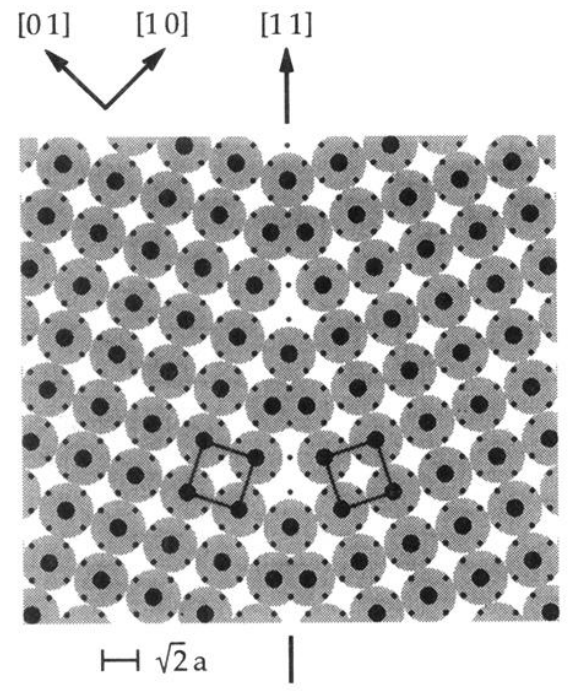

FIG. 1. Two-dimensional bicrystal on a square lattice with a high-angle symmetric tilt boundary in the [1 1 ] direction ("unrelaxed" state). The figure shows the sites of the square lattice (dots), the occupied sites (solid circles), and the associated "atomic" hard-core disks with diameter $\sqrt{5} a$ (gray circles). Also shown are the unit cells of crystalline $(\sqrt{5} \times \sqrt{5}) R \pm \arctan \frac{1}{2}$ domains of type "+" (left) and "-" (right). 

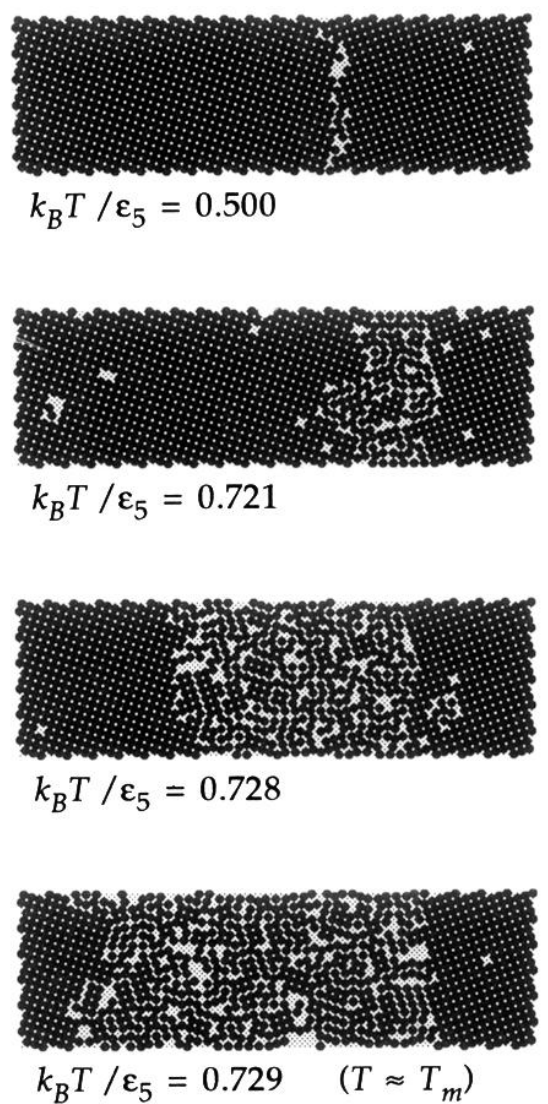

FIG. 2. Typical Monte Carlo microconfigurations of a bicrystal model of size $L \times M=100 \times 30$ (6000 lattice sites) after equilibration at the temperature $T$ shown below each picture $\left(\mu / \varepsilon_{5}=-1.5\right)$. Hard-core disks are indicated by solid circles. 